\title{
Augmented Reality in der Fabrik
}

\author{
Wie der Assistent der Zukunft aussehen könnte
}

$\checkmark$ Immersive Nutzendemonstration im ZIP4.0 bereitet die Nutzer bereits im Vorfeld auf den zukünftigen Einsatz vor.

$\checkmark$ Produktivitätssteigerung mittels prozessindividuellen AR-Anwendungen realisieren.

$\checkmark$ Generierung von Mehrwerten für das Unternehmen durch den effizienten Einsatz digitaler Assistenzsysteme in der Werkhalle.

Im Zentrum Industrie 4.0 Potsdam (ZIP 4.0) kann diese Frage individuell und ohne großen Aufwand beantwortet werden. Mehr noch, mit Hilfe der hybriden Simulationsumgebung ist die Interaktion mit dem AR-Gerät durch den Akteur innerhalb von Fertigungsprozessen möglich. So kann nicht nur der Nutzen demonstriert, sondern auch durch den tatsächlichen Einsatz innerhalb der realitätsnahen Prozessabbildung die Akzeptanz für die spätere Nutzung geschaffen werden.

Augmented Reality (AR) ist ein Baustein aus dem Portfolio von Industrie 4.0 und bietet mit der Informationsbereitstellung innerhalb des Sichtfeldes auf den assoziierten Betrachtungsgegenstand Potenziale für die Prozessverbesserung in der Fertigung. Allerdings ist es bisher nur unzureichend gelungen, die theoretischen Potenziale von AR-basierten Lösungen auf die Straße bzw. in die Fabrik zu bekommen. Das liegt unter anderem darin begründet, dass eine erfolgreiche Einführung einer derartigen Lösung nicht nur die Beschaffung eines AR-Gerätes umfasst. Sie bedarf ebenso einer geeigneten Prozessintegration mit der Anbindung an bestehende Anwendungssystem zwecks Datenaustausch als auch der Akzeptanz durch den Nutzer. Alle drei Aspekte müssen innerhalb von AR-Projekten ausreichend berücksichtigt werden. Das Zentrum Industrie 4.0 Potsdam

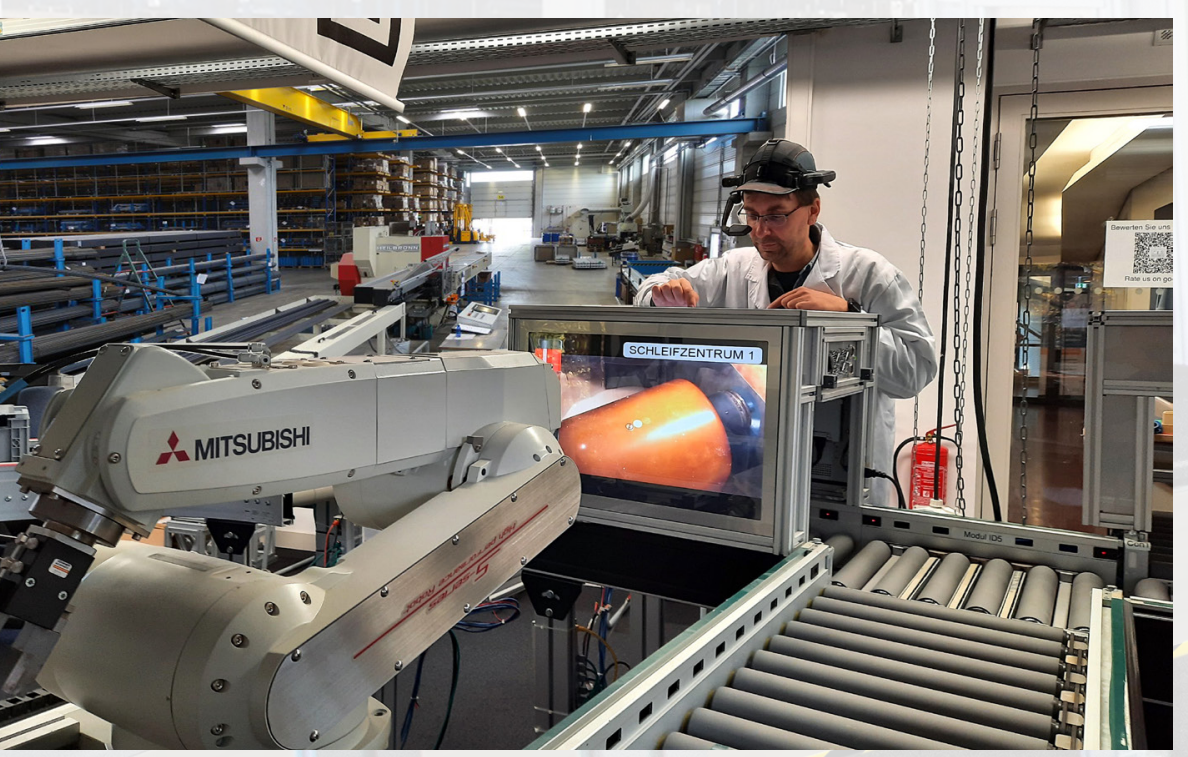

bietet mit der hybriden Simulationsumgebung daher ein nützliches Instrument der Erkenntnisgewinnung.

\section{Eine wirkungsvolle Testumgebung}

Die hybride Simulationsumgebung des Zentrums Industrie 4.0 Potsdam ist eine neuartige Modellfabrik, in der sowohl virtuelle als auch reale Elemente der Fertigung miteinander interagieren. Alle Elemente sind als Industrie 4.0-fähige Produktionsobjekte konfigurierbar und können einfach zu Produktionsprozessen verknüpft werden. Ergebnis ist ein Test- und Evaluationswerkzeug, welches mit hoher Immersion Szenarien und Prozesse aus dem Produktionskontext nachbildet und Erkenntnisse zu Eignung und konkreter Ausgestaltung von Technologien und Organisationskonzepten für die Werkhalle generiert. Da die Originalakteure einbezogen werden, ergeben sich besondere Anforderungen in der Praxis. Ebenso wird die Akzeptanz gesteigert, da zukünftige Lösungen innerhalb eines praxisnahen Produktionsprozessmodells tatsächlich erlebbar und damit zum Erlebnis werden.

\section{AR-Lösungen zum Anfassen}

In der hybriden Simulationsumgebung stehen AR-basierte Elemente für die Gestaltung unterschiedlicher Anwendungsfälle bereit. Dies umfasst eben nicht nur das AR-Gerät sondern ebenso dessen Anbindung an die betrieblichen Anwendungssysteme. So ist es möglich, den Technologiebaustein auf schnelle und einfache Weise in Produktionsszenarien einzubinden und potenzielle Vorteile aufzuzeigen. Eingebunden 


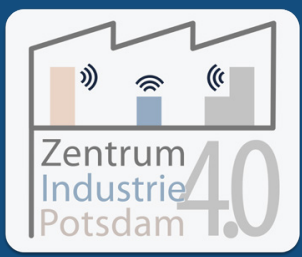

in den im Prozessablauf nutzen Akteure in der Modellfabrik AR als reales Hilfsmittel der Problemlösung. So stellt der Werker beispielsweise fest, dass diese Art des gezielten Informationszugangs seine Arbeit wahrhaftig erleichtern kann. Kombiniert mit einer Sprachsteuerung ermöglichen die AR-Brillen das handfreie Arbeiten. Die Verknüpfung mit bestehender Schutzausrüstung fördert zusätzlich die positive Wahrnehmung. Neben der Nutzendemonstration und -evaluierung ergeben sich durch Beobachtungen des Nutzerverhaltens in den Szenarien wertvolle Erkenntnisse hinsichtlich der Usabilily, die einen wesentlichen Akzeptanzfaktor darstellt.

\section{Die richtige AR-Lösung}

Für Fertigung erscheinen insbesondere ARBrillen als eine vielversprechende Variante der AR-Anwendung. Sie ermöglichen das handfreie Arbeiten und können gleichzeitig Schutzfunktionen übernehmen. Aktuell bekanntester Vertreter ist sicher Microsofts HoloLens. Neben dem hohen Preis und der mangelnden Verfügbarkeit scheint dieses Gerät für die industrielle Anwendung in der Werkhalle nur bedingt geeignet. Es bedarf entsprechender AR-Geräte, die mit den rauen Umgebungsbedingungen in der Fabrik zurechtkommen und trotzdem eine geeignete Usability aufweisen. Glücklicherweise bietet der Markt weitere Lösungen und es gilt, die richtige Variante für den individuellen Anwendungsfall bzw. die existierende Prozessumgebung auszuwählen. Das ZIP 4.0 bietet hier ein passendes Werkzeug, beginnend mit der Identifikation geeigneter Use Cases über die Auswahl passender AR-Geräte und deren Integration bis zur Schaffung von mehr Akzeptanz bei den (zukünftigen) Anwendern.

\section{Anwendungsfall Assistierte Wartung}

Für die Nutzendemonstration und Lösungsauswahl bildet die hybride Simulationsumgebung einen typischen Produktionsprozess ab, bestehend aus Maschinen und Werkstückträgern, physischem Materialtransport. Ebenso sind Industrieroboter und Handarbeitsplätze konfiguriert. Die Produktion läuft und die Werker agieren in der Anlage, bedienen Maschinen und planen Arbeitsgänge. Eine unerwartete Störung am Schleifzentrum führt zum Stillstand der Fertigung. Schnellstmöglich muss das Problem behoben werden. An der Maschine ergibt

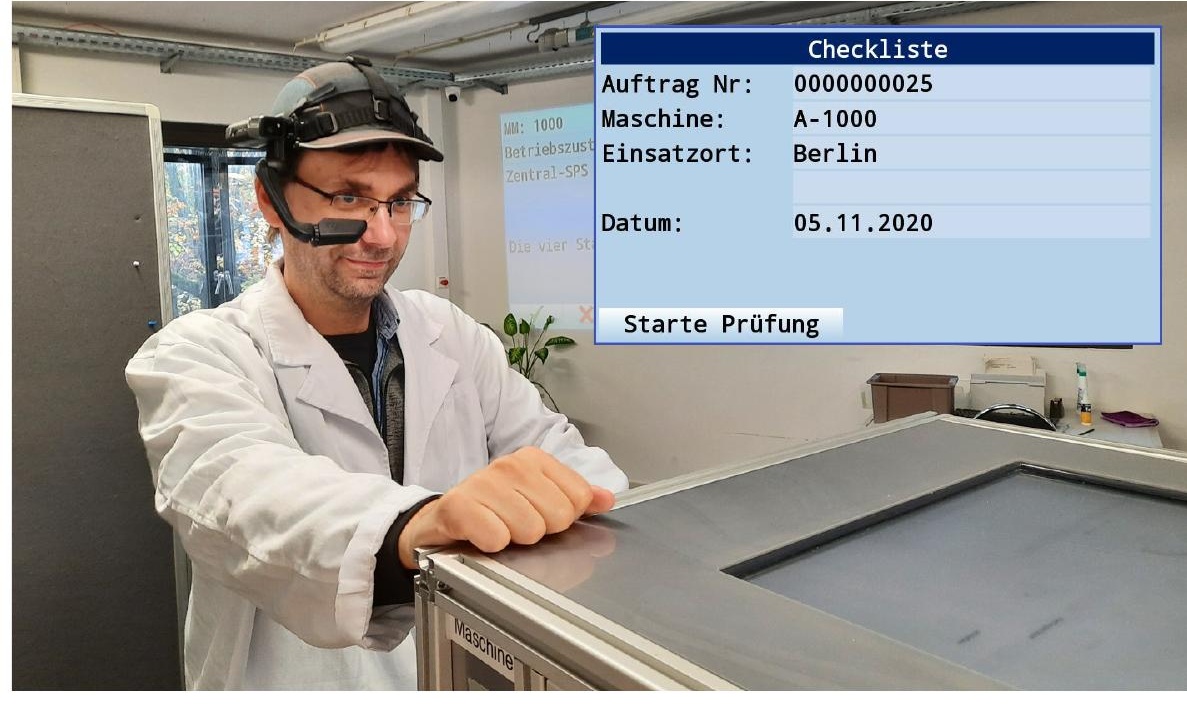

sich ein komplexes Fehlerbild, welches über die routinemäßige Bearbeitung hinausgeht. Die Modellfabrik realisiert drei Szenarien für die Störungsbeseitigung. Ohne Assistenz gelingt es dem Werker nur schwer oder gar nicht, die Fertigung zeitadäquat wieder in Betrieb zu setzen. Er ist auf externe Hilfe angewiesen.

Unter Verwendung eines Tablets bietet das zweite Szenario Zugriff auf Diagnosedaten und -dokumentation. Maßnahmenempfehlungen in Form von Checklisten ergänzen das Informationsangebot. Damit steht dem Werker vor Ort ein erweitertes Fehlerbild zur Diagnose zur Verfügung. Die Störungsbeseitigung gelingt, wird aber durch den ständigen Wechsel von Tablet- und Maschineninteraktion verzögert. Im dritten Szenario behebt der Werker dieses Manko unter Einsatz einer industrietauglichen AR-Brille, die in Kooperation mit dem Praxispartner nxtBase-Technologies an die Maschine bzw. die Backendsysteme der Produktionssteuerung angebunden ist. Per Sprachkommandos arbeitet der Werker eine Wartungsliste ab und dokumentiert seine Tätigkeiten per integrierter Kamera. In kürzester Zeit wird wieder produziert.

Der Anwendungsfall der Assistierten Wartung zeigt mit seinen drei Szenarien, dass digitale Assistenzsystem richtig umgesetzt ein wirkungsvolles Werkzeug für Akteure in der Werkhalle darstellen und für das Unternehmen einen hohen Mehrwert bieten können. Durch die positiven Erfahrungen mit dem Assistenzsystem in der Anlage des ZIP4.0 werden Ängste und Vorurteile der potenzieller Nutzer wirkungsvoll abgebaut und die zukünftige Implementierung erleichtert. 